\title{
Acromegalic Arthropathy
}

\author{
Ariel L. Barkan \\ The University of Michigan Health System, Ann Arbor, \\ Michigan, USA
}

Arthropathy is one of the most frequent clinical complications of acromegaly. At presentation, some $60-70 \%$ of patients have involvement of large peripheral joints (shoulder, knee, hip) and about 50\% have axial arthropathy affecting mainly the lumbar area [1-3]. Elevated circulating and likely autocrine IGF-1 is the driving mechanism leading to joint involvement in this disease [4]. Degenerative osteoarthropathy was detected in $43 \%$ of cats with GH-producing pituitary tumors [5]. Conversely, GH deficiency is a powerful protective mechanism against aging-associated degenerative joint disease [6].

Arthropathy may present as the earliest clinical symptom of acromegaly in a significant proportion of patients [7], and its prevalence and severity worsen with the duration of uncontrolled disease [3].

The pathogenesis of arthropathy in acromegaly is comprised of two mechanisms: initial endocrine and subsequent mechanical changes. The understanding of the bi-phasic nature of arthropathy is important for designing appropriate therapeutic strategies.

Early in the course of the disease, elevated GH and IGF-1 levels promote growth of the articular cartilage and periarticular ligaments. As a result, the cartilage lining thickens and the joint space becomes "congested" with ensuing limitation in the range of movements. At the same time, overgrown ligaments cause laxity of the joint. This stage is likely to occur very early in the course of acromegaly and is fully reversible, as evidenced by the clinical course of arthropathy in patients with hypopituitarism overtreated with GH [8]. If allowed to persist, the altered joint geometry results in repeat intra-articular trauma and exuberant reparative reactions which leads to scar, cyst and osteophyte formation with further worsening of joint geometry. The vicious circle of "trauma-growth-trauma" is thus turned on, and the disease acquires the characteristics and the features of degenerative joint disease. Johanson et al. [9] provided a unique description of 11 surgical specimens from acromegalic patients who had total hip arthroplasty and compared them to the findings in patients with DJD. The extent of osteophytes, synovitis and pitting of the subchondral bone in acromegalic patients were qualitatively similar but more pronounced in acromegaly.

Obviously, when the disease is allowed to progress that far, the changes become irreversible and biochemical control of acromegaly, as evidenced by a normal IGF-1, will have limited, if any, efficacy in improving the clinical status of the patient.
Early studies on the reversibility of joint involvement in acromegaly were uniformly pessimistic. Dons et al. [7] studied 90 patients treated primarily by external pituitary radiotherapy. Five or more years after XRT, only $13 \%$ of patients were unaffected by arthropathy, and it actually became worse in $2 / 3$ of the group. These results are not surprising as XRT has a very slow effect on GH hypersecretion and limited efficacy in terms of normalizing plasma IGF-1. Surgical series were no more effective. Whereas Lacks et al. [10] provided anecdotal evidence of resolution of arthropathy in 2 patients after pituitary surgery, Roelfsema et al. [11] had less encouraging results. In a series of 60 patients treated by surgery, plasma GH fell to "normal" in 62\%. Despite that seemingly salutary result, resolution of arthropathy was "seldom seen" even after a mean follow-up of 3.3 years. Part of this discouraging outcome might have been due to the already irreversible nature of the joint disease, but persistently high IGF-1 levels were likely maintained, even in some patients with plasma GH below 2.5 microgram $\mu / \mathrm{L}$, perpetuating the progression of arthropathy.

Layton et al. [3] were the first to examine the clinical response of acromegalic arthropathy to the potent GH-lowering agent, octreotide. A mild-to-moderate improvement in symptoms, estimated functional ability and in crepitus occurred in 8 of 9 patients. Colao et al. $[12,13]$ pioneered the technique of ultrasound assessment of articular cartilage thickness in acromegaly. They have shown that articular cartilage almost doubles in thickness in all major joints (shoulder, knee, wrist) in active acromegaly and shrinks (but does not normalize) in patients with inactive disease [12]. Treatment with octreotide decreased cartilage thickness primarily in nonweight bearing joints and effected clinical improvement. In a subsequent study, the same group examined the effects of lanreotide therapy. Again, a decrease in cartilage thickness and marked clinical improvement were observed in virtually all patients in parallel with normalization of plasma IGF-1 [13]. Interestingly, the effect sometimes required as long as 12 months of therapy.

Thus, aggressive therapy of acromegaly aimed at normalization of plasma IGF-1 is a prerequisite for

Address correspondence to: Ariel L. Barkan, MD, University of Michigan Medical Center, 3920 Taubman Center, Box 0354, Ann Arbor, MI 48109-0354, USA. Tel: (734) 615-6964; Fax: (734) 936-9240; E-mail: abarkan@umich.edu. 
successful control of arthropathy. This often requires multiple therapeutic modalities including surgery, pharmacotherapy and, in some cases, pituitary irradiation.

Obviously, not all patients will obtain sufficient symptomatic relief from normalization of their GH/IGF-1 milieu. Any residual morbidity has to be dealt with using traditional methods: physical therapy, NSAIDs, local steroid injections or, in advanced cases, surgical interventions (including joint replacement).

To obtain the best chance of sparing the patient the agony and the disability of acromegalic arthropathy, the disease should be optimally diagnosed as early as possible and treated aggressively from the outset. Normalization of plasma IGF-1 is currently the best available monitoring tool, although means of assessment of the magnitude of autocrine IGF-1 production would be highly desirable.

\section{References}

1. Detenbeck LC, Tressler HA, O'Duffy JD, Randall RV. Peripheral joint manifestations of acromegaly. Clin Orthop 1973;91:119127.

2. Podgorski M, Robinson B, Weissberger A, Stiel J, Wang S, Brooks PM. Articular manifestations of acromegaly. Aust $N$ $Z$ J Med 1988;18:28-35.

3. Layton MW, Fudman EJ, Barkan A, Braunstein EM, Fox IH. Acromegalic arthropathy. Characteristics and response to therapy. Arthritis Rheum 1988;31:1022-1027.

4. Okazaki K, Jingushi S, Ikenoue T, Urabe K, Sakai H, Ohtsuru A, Akino K, Yamashita S, Nomura S, Iwamoto Y. Expression of insulin-like growth factor 1 messenger ribonucleic acid in developing osteophytes in murine experimental osteoarthritis and in rats inoculated with growth hormone-secreting tumor. Endocrinology 1999;140:4821-4830.

5. Peterson ME, Taylor RS, Greco DS, Nelson RW, Randolph JF, Foodman MS, Moroff SD, Morrison SA, Lothrop CD. Acromegaly in 14 cats. $J$ Vet Intern Med 1990;4:192-201.

6. Bagge E, Eden S, Rosen T, Bengtsson BA. The prevalence of radiographic osteoarthritis is low in elderly patients with growth hormone deficiency. Acta Endocrinol 1993;129:296300 .

7. Dons RF, Rosselet P, Pastakia B, Doppman J, Gorden P. Arthropathy in acromegalic patients before and after treatment: A long-term follow-up study. Clin Endocrinol 1988;28:515-524.

8. Chipman JJ, Attanasio AF, Birkett MA, Bates PC, Webb S, Lamberts SW. The safety profile of GH replacement therapy in adults. Clin Endocrinol 1997;46:473-481.

9. Johanson NA, Vigorita VJ, Goldman AB, Salvati EA. Acromegalic arthropathy of the hip. Clin Orthop 1983;173:130-139.

10. Lacks S, Jacobs RP. Acromegalic arthropathy: A reversible rheumatic disease. J Rheumatol 1986;13:634-636.

11. Roelfsema F, van Dulken H, Frolich M. Long-term results of transsphenoidal pituitary microsurgery in 60 acromegalic patients. Clin Endocrinol 1985;23:555-565.

12. Colao A, Marzullo P, Vallone G, Marino V, Annecchino M, Ferone D, De Brasi D, Scarpa R, Oriente P, Lombardi G. Reversibility of joint thickening in acromegalic patients: An ultrasonography study. J Clin Endocrinol Metab 1998;83:21212125.

13. Colao A, Marzullo P, Vallone G, Giaccio A, Ferone D, Rossi E, Scarpa R, Smaltino F, Lombardi G. Ultrasonographic evidence of joint thickening reversibility in acromegalic patients treated with lanreotide for 12 months. Clin Endocrinol 1999;51:611618. 